\title{
Gas Flows in Galaxies: the Relative Importance of Mergers and Bars.
}

\author{
Sara L. Ellison ${ }^{1}$, David R. Patton ${ }^{2}$, Preethi Nair ${ }^{3}$, Luc Simard ${ }^{4}$, \\ J. Trevor Mendel ${ }^{1}$, Alan W. McConnachie ${ }^{4}$ and Jillian M. Scudder ${ }^{1}$ \\ ${ }^{1}$ Department of Physics and Astronomy, University of Victoria, Victoria, British Columbia, \\ V8P 1A1, Canada \\ ${ }^{2}$ Department of Physics \& Astronomy, Trent University, 1600 West Bank Drive, Peterborough, \\ Ontario, K9J 7B8, Canada \\ ${ }^{3}$ INAF-Astronomical Observatory of Bologna, Via Ranzani 1, 40127 Bologna, Italy. \\ ${ }^{4}$ National Research Council of Canada, Herzberg Institute of Astrophysics, 5071 West Saanich \\ Road, Victoria, British Columbia, V9E 2E7, Canada
}

\begin{abstract}
Galaxy-galaxy interactions and large scale galaxy bars are usually considered as the two main mechanisms for driving gas to the centres of galaxies. By using large samples of galaxy pairs and visually classified bars from the Sloan Digital Sky Survey (SDSS), we compare the relative efficiency of gas inflows from these two processes. We use two indicators of gas inflow: star formation rate (SFR) and gas phase metallicity, which are both measured relative to control samples. Whereas the metallicity of galaxy pairs is suppressed relative to its control sample of isolated galaxies, galaxies with bars are metal-rich for their stellar mass by 0.06 dex over all stellar masses. The SFRs of both the close galaxy pairs and the barred galaxies are enhanced by $\sim 60 \%$, but in the bars the enhancement is only seen at stellar masses $\mathrm{M}_{\star}>10^{10} \mathrm{M}_{\odot}$. Taking into account the relative frequency of bars and pairs, we estimate that at least three times more central star formation is triggered by bars than by interactions.
\end{abstract}

Keywords. galaxies: abundances, galaxies: interactions

\section{Introduction}

The relative importance of external versus internal processes in galaxy evolution is an ongoing debate in astronomy. One contemporary example of this debate was discussed by Chris Conselice at this meeting, namely the question of whether galaxy mass (an intrinsic property) or environment (external influence) has a larger impact on a galaxy's evolution. In this contribution, we will investigate what mechanism is most important for triggering gas flows to galactic centres, considering both the internal process of bar formation and the external effect of galaxy-galaxy interactions. Both of these processes have been well-documented in the literature to trigger star formation due to the inflow of gas through tidal torques and angular momentum loss (e.g. Martinet \& Friedli 1997; Barton et al. 2000; Nikolic et al. 2004; see also the contributions by Perez and Di Matteo in these proceedings). However, a direct comparison of the two mechanisms requires a large, homogeneous dataset and consistent technical analyses.

We note that bars can themselves be formed during interactions, so the two processes are not entirely independent. However, only 4 of the bars in our sample appear to be currently undergoing a strong interaction, consistent with typical merger fractions at this low redshift. We therefore consider that bars represent a much more extended phase in the galaxy's history than a fly-by or merger. Our comparison is therefore also one of timescales, comparing the effect of a close encounter which is short-lived but potentially 
dramatic, and the longer-lived bar phase (which may be either interaction-induced or secular).

\section{Sample and Analysis}

We have selected a sample of spectroscopic galaxy pairs from the SDSS DR7 with small velocity differences $(\Delta V<300 \mathrm{~km} / \mathrm{s})$ and projected separations $\left(r_{p}<30 \mathrm{kpc}\right)$. Similarly, we have used a sample of visually classified bars from the SDSS DR 4 compiled by Nair \& Abraham (2010a). We further require that reliable spectroscopic SFRs and gas phase metallicities are available, resulting in samples of bars and pairs that contain 311 and 431 galaxies respectively. Details of the full sample selection can be found in Ellison et al. (2008a, 2010, 2011) and Patton et al. (2011). An important component of our analysis is the construction of control samples matched simultaneously in stellar mass and redshift from a pool of galaxies without close companions (in the case of the galaxy pair control sample) or unbarred galaxies (in the case of the barred galaxy sample). The matching is done iteratively and without replacement until a Kolmogorov-Smirnov test drops below $30 \%$ for either a comparison of the masses or redshifts. Matching multiple control galaxies to each test galaxy greatly reduces the statistical uncertainties in the properties of the control sample.

To quantify the effect of gas inflows, we use two metrics: SFR and metallicity. Montuori et al. (2010) have shown that gas inflows simultaneously trigger central star formation and result in an initial dilution of the gas phase metallicity. After the starburst is complete, the galactic interstellar medium (ISM) is gradually enriched by the nucleosynthetic products of the triggered star formation. Studying SFRs and metallicity therefore not only provides evidence of gas inflows, but also a timescale on which these processes occur. To quantify changes in the SFR and metallicity, the mass-SFR and mass-metallicity relations of the bar/pair control samples are fit, such that we can predict the expected SFR and metallicity of a galaxy at a given stellar mass. These fits are performed on the fibre quantities which, in combination with the redshift matching, mitigates the impact of aperture effects (the SDSS fibres cover only the inner few kpc of the galaxies). The actual values of SFR and metallicity in the bars/pairs are compared with the predicted values (for their stellar mass) and an offset $(\Delta)$ is calculated from the difference.

\section{Results: Galaxy Pairs}

Previous studies of close galaxy pairs have shown that galaxy interactions result in low metallicities for their luminosity (Kewley et al. 2006) and high SFRs (e.g. Barton et al. 2000; Nikolic et al. 2004, amongst many others). However, the large samples afforded by SDSS allow us to tease apart the dependences of these offsets as a function of higher order properties. These results have already been published in the literature and we only briefly review the results of our group.

Considering first the metallicity. Ellison et al. (2008a) showed that about $50 \%$ of the offset in the luminosity-metallicity relation in pairs is due to increased luminosity. Considering the mass-metallicity relation, it was shown that pairs are metal-poor for their mass by only 0.03 dex, supporting the interpretation that interactions are experiencing metallicity dilution due to gas inflows.

Turning now to SFRs, Ellison et al. (2008a) showed that triggered SFRs are highest in the major (more equal mass) interactions. Furthermore, Ellison et al. (2010) demonstrated that the triggered SFR depends strongly on environment. Galaxy pairs in low density environments are enhanced by $60 \%$, but no SFR enhancement is seen in pairs in 
the highest densities. Asymmetries in galaxy morphologies exist in the closest separation pairs in all environments, indicating that although interactions occur over a wide range of environments, star formation is triggered preferentially at low densities. This is likely due to the prevalence of gas-rich galaxies in low density environments, which have a ready supply of fuel for star formation. Finally, the triggered star formation is central as evidenced by much bluer colours in the bulge (Ellison et al. 2010) and fibre (Patton et al. 2011) colours. Global colours are much less affected and disks do not show any colour change at close separations.

\section{Results: Galaxy Bars}

Like galaxy pairs, barred galaxies show an increase in their SFRs (at a given stellar mass) relative to the unbarred control sample by $\sim 60 \%$. However, as shown in the left panel of Figure 1, whereas the pairs' SFR enhancement is seen at all stellar masses, in barred galaxies it is only seen for $\mathrm{M}_{\star}>10^{10} \mathrm{M}_{\odot}$. This mass threshold has been shown by Nair \& Abraham (2010b) to correspond to the fairly rapid transition between the weakly barred, low mass, late-type spirals and the more strongly barred, higher mass early types.

In contrast to galaxy pairs, barred galaxies show an enhanced metallicity for a given stellar mass by $\sim 0.06$ dex (Figure 1, right panel). This indicates that bars are relatively long-lived and the star formation is likely to be extended in time with multiple bursts (see also contributions by Perez and Robert in these proceedings). Interestingly, the metal enhancement is seen even at $M_{\star}<10^{10} M_{\odot}$ where there is no enhanced star formation. This can be explained by an early, but short-lived, period of enhanced star formation at low mass, such that we see the chemical enrichment, but the actual starburst is long passed. This interpretation is supported by the models of Combes \& Elmegreen (1993) who find that high mass galaxies are able to grow their bars over a longer period of time, and to greater extents than lower mass galaxies.

It has been recently demonstrated that the mass-metallicity relation for the general star-forming galaxy population is itself modulated by SFR, such that galaxies with higher SFRs tend to have lower metallicities (Ellison et al. 2008b). Mannucci et al. (2010)
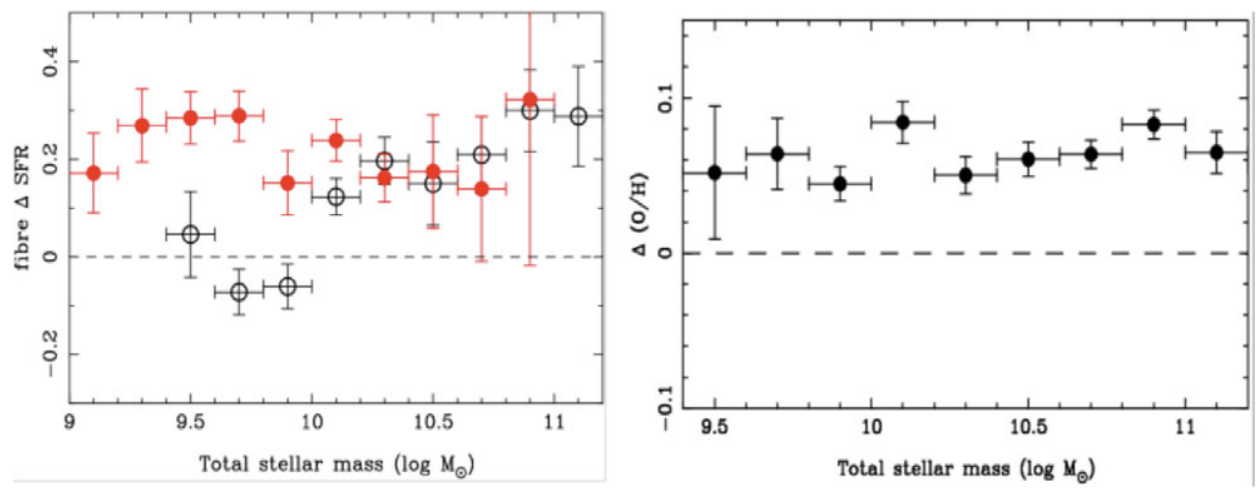

Figure 1. Left panel: the enhancement in fibre star formation rate ( $\Delta$ SFR) as a function of stellar mass for bars and pairs. Enhancements are relative to samples matched in mass and redshift. Solid points show the enhancement in close pairs with $\Delta V<300 \mathrm{~km} / \mathrm{s}$ and $r_{p}<30 \mathrm{kpc}$. Open points show the enhancement for barred galaxies in the visually classified sample of Nair \& Abraham (2010a). Right panel: enhancements in metallicity in barred galaxies relative to a control sample of unbarred galaxies. 
and Lara-Lopez et al. (2010) have even suggested a fundamental relation between SFR, mass and metallicity in star-forming galaxies that can be fit with a plane. Interestingly, barred galaxies do not follow this general trend, since they have both enhanced SFRs and higher metallicities for their mass, and would therefore presumably be outliers on the 'fundamental relation'.

Finally, to compare the relative impact of bars and interactions on triggered star formation $\left(\epsilon_{b / p}\right)$ we must consider the relative fraction of bars and pairs in the parent galaxy sample $\left(f_{b} / f_{p}\right)$ and the fraction of bars and pairs that made it into our starforming (emission line selected) sample $\left(f_{b, \star} / f_{p, \star}\right)$, i.e.

$$
\epsilon_{b / p}=\frac{f_{b}}{f_{p}} \times \frac{f_{b, \star}}{f_{p, \star}} \times \frac{10^{\Delta S F R_{b}}}{10^{\Delta S F R_{p}}} .
$$

We find $\epsilon_{b / p} \sim 3$. However, this is likely to be a lower limit since visually classified barred samples in the optical tend to yield relatively low bar fractions (e.g. relative to the IR). We therefore conclude that bars contribute at least 3 times more to the centrally triggered star formation than interactions. For gas flows, internal processes seem to outweigh external mechanisms.

\section{References}

Barton, E. J., Geller, M. J., \& Kenyon, S. J., 2000, ApJ, 530, 660

Combes, F. \& Elmegreen, B. G., 1993, A\&BA, 271, 391

Ellison, S. L., Patton, D. R., Simard, L., \& McConnachie, A. W., 2008a, AJ, 135, 1877

Ellison, S. L., Patton, D. R., Simard, L., \& McConnachie, A. W., 2008b, ApJ, 672, L107

Ellison, S. L., Patton, D. R., Simard, L., McConnachie, A. W., Baldry, I. K., \& Mendel, J. T., 2010, MNRAS 407, 1514.

Ellison, S. L., Nair, P., Patton, D. R., Simard, L., Scudder, J., \& Mendel, J. T., 2011, MNRAS, submitted

Kewley, L. J., Geller, M. J., \& Barton, E. J., 2006, AJ, 131, 2004

Lara-Lopez, M. A., Cepa, J., Bongiovanni, A., Perez Garcia, A. M., Ederoclite, A., Castaneda, H., Fernandez Lorenzo, M., Povic, M., \& Sanchez-Portal, M., 2010, A\& A, 521, L53

Mannucci, F., Cresci, G., Maiolino, R., Marconi, A., \& Gnerucci, A., 2010, MNRAS, 408, 2115 Martinet, L. \& Friedli, D., 1997, AESA, 323, 363

Montuori, M., Di Matteo, P., Lehnert, M. D., Combes, F., \& Semelin, B., 2010, A\& $A, 518,56$

Nair, P. B. \& Abraham, R. G., 2010a, ApJS, 186, 427

Nair, P. B. \& Abraham, R. G., 2010b, ApJ, 714, L260

Nikolic, B., Cullen, H., \& Alexander, P., 2004, MNRAS, 355, 874

Patton, D. R., Ellison, S. L., Simard, L., McConnachie, A. W., \& Mendel, J. T., 2011, MNRAS, in press 